\title{
Relación de la actividad física con los factores de riesgo cardiovascular en ciudadanos adultos de Melilla
}

\author{
Relationship of the influence of physical activity with \\ cardiovascular risk factors in adult citizens of Melilla
}

\author{
Elisabet Fernández Gómez ${ }^{1}$, María Angustias Sánchez-Ojeda, ${ }^{1, *}$, Adelina Martín-Salvador ${ }^{1}$ y Carmen Enrique Mirón ${ }^{2}$
}

\author{
1 Departamento de Enfermería. Universidad de Granada. Facultad de Ciencias de la Salud. Campus de Melilla (España). \\ 2 Departamento de Quimica Inorgánica. Universidad de Granada. Facultad de Ciencias de la Educación y del Deporte. Campus de Melilla (España).
}

\begin{abstract}
Resumen: La actividad física influye positivamente en la salud de las personas, teniendo un papel fundamental en la prevención, tratamiento, control y rehabilitación de las enfermedades crónicas no transmisibles. El objetivo del estudio es analizar la relación entre la práctica de actividad física y los factores de riesgo cardiovascular. Estudio transversal y correlacional mediante un cuestionario con una muestra de 160 participantes. Los resultados muestran que el $70 \%$ de la muestra manifiesta no hacer prácticamente actividad física frente a un $33 \%$ que afirma realizar una actividad física moderada/alta. De las variables relacionadas con el riesgo cardiovascular sexo, glucemia y tensión arterial sistólica se encuentran asociadas con el grado de actividad física de forma significativa $(\mathrm{p}<.05)$. Es fundamental reforzar la educación sanitaria a toda la población para mantener una adecuada actividad física y otros estilos de vida saludables que repercutan en la disminución de las enfermedades crónicas prevenibles.

Palabras Clave: Actividad física, estilo de vida saludable, enfermedades crónicas, factores de riesgo cardiovasculares.
\end{abstract}

Abstract: Physical activity has a positive influence on people's health, playing a key role in the prevention, treatment, control and rehabilitation of chronic non-communicable diseases. The aim of the study is to analyse the relationship between the practice of physical activity and cardiovascular risk factors. Cross-sectional and correlational study using a questionnaire with a sample of 160 participants. The results show that $70 \%$ of the sample stated that they did not do much physical activity compared to $33 \%$ who stated that they did moderate/high levels of physical activity. Of the variables related to cardiovascular risk, sex, glycemia and systolic blood pressure are significantly associated with the degree of physical activity $(\mathrm{p}<.05)$. It is essential to strengthen health education for the entire population in order to maintain adequate physical activity and other healthy lifestyles that will have an impact on reducing preventable chronic diseases.

Key Words: Motor Activity, Healthy Lifestyle, Chronic Disease, Cardiovascular Risk Factors.

\section{Introducción}

Entre el año 1975 y el 2016, la prevalencia mundial de obesidad casi se ha triplicado. Una de las principales causas de sobrepeso y obesidad a nivel mundial es un descenso en la actividad física debido a las formas de trabajo cada vez más sedentarias, la diversidad en transporte y la creciente urbanización (Organización Mundial de la Salud, 2018a).

El sedentarismo en Europa es muy elevado y, en nuestro país ocupa la preocupante cuarta posición, siendo el 60\% de los españoles adultos sedentarios (OMS, 2019).

Actualmente, en España más del 50\% de la población mayor de 18 años presenta exceso de peso, si a esto le sumamos la prevalencia de sedentarismo, más de la mitad de la población española presenta algún factor de riesgo para padecer alguna enfermedad crónica (Ministerio de Sanidad, Consumo y Bienestar Social, 2017).

Según la Encuesta Europea de Salud en España (EESE, 2014), Melilla es la ciudad española con mayor sobrepeso

Dirección para correspondencia [Correspondence address]: María Angustias Sánchez-Ojeda. C/Santander, 152005 Melilla (España). E-mail: maso@ugr.es
(46.80\%), frente a Madrid (31,89\%) que es la que presenta el menor índice de sobrepeso. Asimismo, es la segunda Comunidad Autónoma de España con mayor porcentaje de sedentarismo (56.79\%) en tiempo de ocio (Ministerio de Sanidad, Consumo y Bienestar Social, 2017) y una de las que exhibe un mayor parque automovilístico por superficie según la Dirección General de Tráfico (DGT, 2018).

La evidencia científica ha demostrado a lo largo de los años que realizar actividad física influye positivamente en la salud de las personas, siendo su papel fundamental en la prevención, tratamiento, control y rehabilitación de numerosas enfermedades crónicas no transmisibles (ECNT) (Mahecha, 2019).

Las ECNT se definen como aquellas enfermedades que comportan una gran repercusión sobre el estilo de vida del paciente; se trata de un proceso incurable y por tanto, implica vivir toda la vida con dicha enfermedad (Martos, Pozo y Alonso, 2010). Las ECNT más importantes se pueden resumir en cuatro enfermedades más prevalentes y son las cardiovasculares, algunos tipos de cáncer, enfermedades respiratorias y pulmonares y trastornos metabólicos como 
la diabetes tipo 2 (Orozco-Gómez y Castiblanco-Orozco, 2015).

Estas ECNT son producidas por el cambio de vida de nuestra sociedad globalizada, que ha ido instaurando en nuestras vidas determinados comportamientos inadecuados que son modificables y que aumentan el riesgo de padecerlas. Los factores de riesgo más importantes son el consumo de tabaco, las dietas no saludables, el uso nocivo del alcohol y el sedentarismo. Todos estos factores de riesgo son determinantes sociales que se pueden evitar y prevenir; y son los causantes de las numerosas cifras de sobrepeso, obesidad, diabetes, hipertensión arterial y colesterol elevado (Tobar y Marchiori, 2018).

Debido a los avances tecnológicos que ha aumentado el ocio digital en los niños y los jóvenes, y que han facilitado las labores del trabajo diario, así como a las responsabilidades laborales y familiares en los adultos y en general a la desmotivación de la población a realizar actividad física, el sedentarismo ha aumentado a nivel mundial. La Organización Mundial de la Salud (OMS) ha catalogado al sedentarismo como el cuarto factor de riesgo en lo que respecta a la mortalidad mundial (OMS, 2018b).

Se define a una persona sedentaria como la que no realiza al menos 30 minutos de actividad física moderada durante la mayoría de los días de la semana (Crespo-Salgado, DelgadoMartín, Blanco-Iglesias y Adecoa-Landesa, 2015). Las consecuencias derivadas del sedentarismo son muy variadas. Así, es responsable del $6 \%$ de la enfermedad arterial coronaria, el $7 \%$ de la diabetes tipo 2 , el $10 \%$ del cáncer de mama, el $10 \%$ del cáncer de colon y el $9 \%$ de la mortalidad prematura (Arocha, 2019).

A lo largo del tiempo y en las evidencias encontradas, existen numerosas publicaciones que recomiendan un determinado tiempo de ejercicio físico al día o a la semana para que este influya positivamente en la salud. En 1995, el American College of Sports Medicine indicaba que las personas adultas debían realizar al menos 30 minutos de actividad física de intensidad moderada durante 5 días a la semana (Pate et al., 1995).

Unos años después, la OMS actualizó esta recomendación e indicó que una persona adulta tendría que acumular 150 minutos de actividad moderada o 75 minutos de actividad vigorosa por semana, o una combinación equivalente de actividades moderadas y vigorosas (OMS, 2010).

La prescripción de ejercicio físico es útil para alcanzar beneficios para la salud, como son la mejoría en el peso corporal, tensión arterial (TA), glucemia y triglicéridos. Dichos beneficios se observan en ambos sexos y es superior cuanto mayor es el volumen o la intensidad del ejercicio (Subirats-Bayego, Subirats-Vila y Soteras- Martínez, 2012).

Es importante concienciar a la población para mantener o conseguir unos hábitos de vida saludables y así prevenir numerosas enfermedades. Esta promoción de hábitos saludables, especialmente de la actividad física, es una labor primordial de los profesionales de la educación y de la salud, por lo que es esencial que estos profesionales estén formados en la materia.

Las intervenciones para promocionar la actividad física pueden ayudar a aumentar los niveles de esta (Bonilla-Arena y Sáez-Torralba, 2014). Lo primordial es realizar algún ejercicio físico para mejorar la condición física, y si lo que se pretende es mejorar o reducir los factores de riesgo de padecer alguna enfermedad cardiovascular como la hipertensión o hipercolesterolemia, es necesario no disminuir las recomendaciones de actividad física (Mahecha, 2019).

La educación para la salud es una disciplina que surge ante la necesidad de dispensar a las personas los métodos necesarios para mejorar su salud, modificando así, las posibles conductas de riesgo, con el fin de alcanzar niveles de salud óptimos.

La finalidad de este estudio se centra en analizar la relación entre la realización de actividad física y los factores de riesgo cardiovasculares en personas de la ciudad Autónoma de Melilla.

\section{Material y método}

\subsection{Diseño}

Estudio transversal, descriptivo y correlacional con ciudadanos melillenses mediante un cuestionario ad hoc con variables socio-demográficas, grado de actividad física y variables sobre el estado de salud.

\subsection{Muestra}

La captación de los participantes en el estudio se realizó mediante muestreo probabilístico quedando conformada la muestra por un total de 160 sujetos, con una edad media de 49.6 años (DT 17.9), de los cuales un tercio (31.3\%) fueron hombres y el $68.8 \%$ fueron mujeres. El $63.8 \%$ de los participantes son de religión cristiana y el $35.6 \%$ pertenece a musulmanes y el $0.6 \%$ practican otras religiones. El $72.6 \%$ disponen de régimen público de seguridad social, el $13.1 \%$ a la sanidad privada y el $14.4 \%$ refiere no ser residente en la ciudad (Tabla 1). 
Tabla 1. Descripción de la muestra. Variables sociodemográficas.

\begin{tabular}{lll}
\hline Variables & & Frecuencia (\%) \\
\hline Sexo & Hombre & $50(31.3)$ \\
& Mujer & $110(68.8)$ \\
\hline Religión & Cristiana & $102(63.8)$ \\
& Musulmana & $57(35.6)$ \\
& Otras & $1(0.6)$ \\
\hline Régimen sanitario & Pública & $116(72.6)$ \\
& Privada & $21(13.1)$ \\
& No residente & $23(14.4)$ \\
\hline
\end{tabular}

\subsection{Análisis estadístico}

El análisis estadístico de los datos se realizó con el paquete informático SPSS 24.0 para Windows. Las variables socio-demográficas se expresaron en frecuencias absolutas y porcentajes. Dado el no ajuste a la normalidad (prueba de Kolmogorov-Smirnov) se utilizaron pruebas no paramétricas para comparar las medias con respecto a la actividad física (U de Mann-Whitney). Para las variables categóricas se utilizaron tablas cruzadas junto al estadístico Chi-cuadrado considerando como valor de significación estadística un valor de $\mathrm{p}<.05$.

\subsection{Procedimiento}

La recogida de datos se llevó a cabo en mesas de salud instaladas en el centro de la ciudad de Melilla durante el mes de marzo del año 2019.
Enfermeros/as y estudiantes de Enfermería recogieron los parámetros de salud: peso y altura, tensión arterial (TA), glucemia aleatoria, enfermedades crónicas, tratamiento actual y grado de realización de actividad física. Además de datos socio-demográficos.

Se informó de la finalidad del estudio y la posibilidad de abandonarlo en cualquier momento, además de la protección de los datos que establece la Ley Orgánica 15/1999, de 13 de diciembre, de Protección de Datos de Carácter Personal. Siendo voluntaria la participación en el estudio.

\subsection{Consideraciones éticas}

La presente investigación se rigió por los principios éticos de la declaración de Helsinki, en la que todos los participantes estuvieron informados del objetivo del estudio, con participación voluntaria y con la firma del consentimiento informado.

\section{Resultados}

En la Tabla 2 se muestran las medias junto a la mediana y moda correspondientes a las variables antropométricas así como los valores medios de glucemia y tensión arterial sistólica y diastólica.

Tabla 2. Variables antropométricas, glucemia y tensión arterial sistólica y diastólica.

\begin{tabular}{llllll}
\hline & Media $(\mathrm{DT})$ & Mediana & Moda & Mínimo & Máximo \\
\hline Peso $(\mathrm{Kg})$ & $71.1(13.6)$ & 70.0 & 70.0 & 44.0 & 129.0 \\
Altura $(\mathrm{cm})$ & $164.4(9.4)$ & 163.0 & 160.0 & 145.0 & 190.0 \\
IMC $\left(\mathrm{kg} / \mathrm{m}^{2}\right)$ & $26.3(4.4)$ & 26.2 & 27.3 & 16.53 & 42.81 \\
Glucemia $(\mathrm{mg} / \mathrm{dL})$ & $116.4(48.7)$ & 99.0 & 110.0 & 69.0 & 367.0 \\
TA Sistólica $(\mathrm{mmHg})$ & $123.9(16.6)$ & 120.0 & 120.0 & 90.0 & 200.0 \\
TA Diastólica $(\mathrm{mmHg})$ & $69.5(9.2)$ & 70.0 & 70.0 & 50.0 & 100.0 \\
\hline
\end{tabular}

En la tabla 3 se presenta la distribución de la muestra participante según índice de masa corporal (IMC), glucemia, TA sistólica y diastólica, y grado de actividad física. Frente a un $34.4 \%$ de participantes con normopeso, el $45 \%$ presenta sobrepeso y un porcentaje nada despreciable (18.7\%) obesidad. Un $22.5 \%$ se encuentra en situación de hiperglucemia frente a un $77 \%$ con valores de glucemia en el rango de la normalidad. En cuanto a la tensión arterial el 92\% de los sujetos muestran valores inferiores a $140 \mathrm{mmHg}$ en sístole y el $98 \%$ inferiores a $90 \mathrm{mmHg}$ en diástole. Finalmente, el 70\% manifiesta no hacer prácticamente actividad física frente a un 33\% que afirma realiza una actividad física media/alta. 
Tabla 3. Distribución según valores de IMC, glucemia, tensión arterial sistólica y diastólica y grado de actividad física.

\begin{tabular}{llc}
\hline Variables & & Frecuencia (\%) \\
\hline IMC & Bajo peso $(<18.5)$ & $3(1.9)$ \\
& Normopeso $(18.5-24.9)$ & $55(34.4)$ \\
& Sobrepeso $(25.0-29.9)$ & $72(45.0)$ \\
& Obesidad I $(30.0-34.5)$ & $26(16.3)$ \\
& Obesidad II $(35.0-39.9)$ & $3(1.9)$ \\
& Obesidad III $(>40.0)$ & $1(0.6)$ \\
\hline Glucemia & Hipoglucemia $(<70 \mathrm{mg} / \mathrm{dL})$ & $1(0.6)$ \\
& Normoglucemia $(70-125 \mathrm{mg} / \mathrm{dL})$ & $123(76.9)$ \\
& Hiperglucemia $(>126 \mathrm{mg} / \mathrm{dL})$ & $36(22.5)$ \\
\hline TA Sistólica & $<140 \mathrm{mmHg}$ & $147(91.9)$ \\
& $>141 \mathrm{mmHg}$ & $13(8.1)$ \\
\hline TA Diastólica & $<90 \mathrm{mmHg}$ & $157(98.1)$ \\
& $>91 \mathrm{mmHg}$ & $3(1.9)$ \\
\hline Actividad & Nula/Baja & $107(66.9)$ \\
física & Media/Alta & $53(33.1)$ \\
\hline
\end{tabular}

De las variables relacionadas con el riesgo cardiovascular solo sexo, glucemia y tensión arterial sistólica se encuentran asociadas con el grado de actividad física de forma significativa $(\mathrm{p}<.05)$. Así, son las mujeres las que en menor medida realizan poca o nula actividad física $(\mathrm{p}=.049)$, los mayores valores en hiperglucemia se presenta entre los participantes que realizan poca o nula actividad física $(\mathrm{p}=.042)$, dato que se confirma al analizar los valores medios de glucemia según el grado de actividad física realizado, presentando medias de 120.65 (DT 48.4) $\mathrm{mg} / \mathrm{dL}$ aquellos participantes que no realizan actividad física mientras que los que sí practican actividad física muestran valores medios de 107.9 (DT 48.8) mg/ $\mathrm{dL} \quad(\mathrm{p}=.020)$. Finalmente, son de nuevo los sujetos que no realizan actividad física los que presentan mayores valores de presión arterial sistólica $(\mathrm{p}=.042)$, tal y como puede apreciarse en la Tabla 4.

Tabla 4. Variables sociodemográficas y variables relacionadas con riesgo cardiovascular en función del grado de actividad física.

\begin{tabular}{|c|c|c|c|c|c|}
\hline \multirow[t]{2}{*}{ Variables } & & \multicolumn{4}{|c|}{ Actividad física } \\
\hline & & Nula/Baja & Media/Alta & Chi-cuadrado & $\mathrm{p}$ \\
\hline \multirow[t]{2}{*}{ Sexo } & Hombre $(\mathrm{n}=50)$ & $28(56.0)$ & $22(44.0)$ & 3.883 & .049 \\
\hline & Mujer $(\mathrm{n}=110)$ & $79(71.8)$ & $31(28.2)$ & & \\
\hline \multirow[t]{3}{*}{ Religión } & Cristiana $(\mathrm{n}=102)$ & $64(62.7)$ & $38(37.3)$ & 4.691 & .096 \\
\hline & Musulmana (n=57) & $43(75.4)$ & $14(24.6)$ & & \\
\hline & Otras $(\mathrm{n}=1)$ & 0 & $1(100)$ & & \\
\hline \multirow[t]{6}{*}{ IMC } & Bajo peso $(n=3)$ & $2(66.7)$ & $1(33.3)$ & 8.420 & .135 \\
\hline & Normopeso $(\mathrm{n}=55)$ & $31(56.4)$ & $24(43.6)$ & & \\
\hline & Sobrepeso $(\mathrm{n}=72)$ & $48(66.7)$ & $24(33.3)$ & & \\
\hline & Obesidad I (n=26) & $22(84.6)$ & $4(15.4)$ & & \\
\hline & Obesidad II $(\mathrm{n}=3)$ & $3(100)$ & 0 & & \\
\hline & Obesidad III (n=1) & $1(100)$ & 0 & & \\
\hline \multirow[t]{3}{*}{ Glucemia } & Hipoglucemia $(\mathrm{n}=1)$ & $1(100)$ & 0 & 6.334 & .042 \\
\hline & Normoglucemia $(\mathrm{n}=123)$ & $76(61.8)$ & $47(38.2)$ & & \\
\hline & Hiperglucemia $(\mathrm{n}=36)$ & $30(83.3)$ & $6(16.7)$ & & \\
\hline \multirow[t]{2}{*}{ TA Sistólica } & $<140 \mathrm{mmHg}(\mathrm{n}=147)$ & $95(64.6)$ & $52(35.4)$ & 4.132 & .042 \\
\hline & $>141 \mathrm{mmHg}(\mathrm{n}=13)$ & $12(92.3)$ & $1(7.7)$ & & \\
\hline \multirow[t]{2}{*}{ TA Diastólica } & $<90 \mathrm{mmHg}(\mathrm{n}=157)$ & $106(67.5)$ & $51(32.5)$ & 1.553 & .213 \\
\hline & $>91 \mathrm{mmHg}(\mathrm{n}=3)$ & $1(33.3)$ & $2(66.7)$ & & \\
\hline
\end{tabular}

Si bien el IMC no presenta una asociación significativa con la realización de actividad física, si se correlaciona de forma positiva con la TAs (Correlación Pearson=.170, $\mathrm{p}=.032$ ), con la TAd (Correlación Pearson=.179, p=.024), y con la glucemia (Correlación Pearson=.257, p=.001), estando esta última relacionada de forma significativa con la TAs (Correlación Pearson=.368, p=.000). 


\section{Discusión}

Más de un tercio (35.3\%) de la población entre 15 y 69 años no alcanza el nivel de actividad física saludable recomendado por la OMS. El incumplimiento de estas recomendaciones es mayor en mujeres (37\% vs. 33.5\%) (Ministerio de Sanidad, Consumo y Bienestar Social, 2017). Los resultados del presente estudio han demostrado que la población de Melilla realiza niveles bajos de actividad física, sobre todo las personas del sexo femenino, dando lugar a que más de la mitad de la población presente exceso de peso.

El efecto protector del ejercicio es evidente, pero para que estos sean óptimos, el ejercicio debe cumplir unos criterios como la regularidad, la intensidad o la duración (Lamotte, 2016).

Según Arteaga, Bustos, Soto, Velasco y Amigo (2010) los hombres realizan mayor ejercicio que las mujeres, se observó que $60 \%$ de los hombres realizaban una actividad física intensa mientras que el $50 \%$ de las mujeres tenían una actividad física insuficiente. En nuestro estudio se observan datos similares, el $44 \%$ de los hombres realizan ejercicio físico medio/alto y el $71.8 \%$ de las mujeres no hacen ejercicio o es de intensidad baja.

El sedentarismo presenta una asociación con factores de riesgo cardiovasculares y metabólicos, afecta de manera perjudicial a los niveles de glucemia (Leiva, 2017) como sucede en este estudio.

Según Leiva (2017) los sujetos altamente sedentarios presentan mayor IMC y obesidad, en el estudio actual no se observa una asociación directa entre la actividad física y el IMC, aunque si se establece una relación indirecta ya que se observa que quienes hacen menos actividad física presentan peores valores en estas variables.

La realización de actividad física resulta determinante para el control de la tensión arterial en pacientes con hipertensión arterial, además disminuye su tasa de mortalidad. En el estudio de Báez et al. (2007) se ha demostrado que la realización de ejercicio físico con regularidad puede disminuir entre 4-10 mmHg la presión arterial sistólica y $8 \mathrm{mmHg}$ la diastólica. En otro estudio realizado a nińos escolares, demostraron que la actividad física resulta muy beneficiosa, mostrando mejoras significativas en la presión arterial sistólica y diastólica (López, Ibáñez y Díaz, 2019).

Vélez-Álvarez y Vidarte-Claros (2016) llevaron a cabo un programa de entrenamiento físico en personas hipertensas, donde se demostró que los pacientes que practicaron ejercicio aeróbico dirigido durante más de 45 minutos tres veces por semana mejoraron sus niveles de LDL, HDL y triglicéridos. $\mathrm{Al}$ igual que en el estudio valenciano que realizaron Caro et al. (2013), en el que demostraron que la realización de ejercicio físico regular influía de manera positiva disminuyendo los niveles de colesterol.
La actividad física presenta una asociación con los indicadores de riesgo cardiovascular TAs y TAd (Arteaga et al., 2010). En este estudio la AF se encuentra asociada de forma significativa con la TAs, siendo menor en aquellas personas que realizan actividad física.

En la revisión sistemática realizada por Quílez-Llopis y García-Galbis (2015) se concluye que el ejercicio aeróbico, el ejercicio de fuerza o la combinación de ambos, llevado a cabo durante 150 minutos a la semana, con una intensidad moderada y con un intervalo menor a 48 horas entre sesiones, mejoran el control glucémico. Igualmente, estudios determinan que la glucosa plasmática posprandial se reduce mediante ejercicio físico aeróbico (Schwaab et al., 2020).

La actividad física también mejora la cognición, especialmente la memoria en deterioro cognitivo leve y la salud psicológica en la demencia (Nuzum et al., 2020). También evidencias actuales determinan que la actividad física está asociada con una disminución del riesgo de cáncer de próstata (Capece et al., 2020).

Realizar actividad física dos veces más de las mínimas recomendaciones previene el riesgo de enfermedad cardiovascular y diabetes tipo 2. Los profesionales de la salud deben estimular a los adultos inactivos a realizar ejercicio y mantenerse activos durante su tiempo libre, y así, conseguir beneficios para la salud (Jo et al., 2020).

Las recomendaciones sobre el fomento del ejercicio físico, además de otros estilos de vida saludables, por parte de los profesionales sanitarios son fundamentales y deben ser constantes, para aumentar el conocimiento y la motivación de la población. Por lo que implementar diversas intervenciones para concienciar a la población de la importancia de llevar unos hábitos de vida saludables, como una dieta adecuada y la realización de ejercicio físico, disminuye las probabilidades de sufrir una enfermedad crónica no transmisible, mejorando los niveles de colesterol, la disminución de la glucemia y de la tensión arterial, tal y como demostraron varios autores en la implementación de un programa de actividad físicorecreativas a mujeres de la tercera edad (Chávez, Fernández, Rodríguez, Gómez y Sánchez, 2017).

Se ha demostrado que las Escuelas de Pacientes, a través de la formación entre iguales, ayudan a incrementar el ejercicio físico y la calidad de la dieta en pacientes con patología crónica, como la diabetes e insuficiencia cardiaca (Danet et al., 2016; Danet et al., 2020).

Es importante promocionar la práctica de actividad física desde la infancia y la adolescencia, ya que el sedentarismo e inactividad física provoca múltiples enfermedades disminuyendo la calidad de vida de la población (Alvarez et al., 2020). 


\section{Conclusiones}

Como se ha demostrado en numerosos estudios, realizar actividad física mejora el estado de salud en general, disminuyendo las cifras de tensión arterial, sobrepeso o las cifras de glucosa en sangre elevada. En este estudio se observa que quienes hacen menos actividad física presentan peores valores en las variables relacionadas con el riesgo cardiovascular. Estos resultados no son generalizables pero sí muestran una cierta tendencia y por tanto habría que profundizar en ello.

Debido al cambio de estilo de vida en la actualidad, la población, en general ha disminuido la actividad física, siendo un hábito fundamental para mantener una buena salud, reducir la tensión arterial, el colesterol, entre otras, y sobre todo para evitar enfermedades crónicas prevenibles. Es necesario concienciar a la población de la importancia de la actividad física como vía para controlar estas variables.

Por lo que, además de una buena educación sanitaria sobre actividad física y otros estilos de vida saludables en colegios, institutos, centros sanitarios, es importante realizar mesas de salud en lugares públicos, donde cualquier persona pueda someterse a la determinación de parámetros de salud y se les pueda dar una información detallada y personalizada.

\section{Referencias}

1. Alvarez-Pitti, J., Casajús-Mallén, J. A., Leis-Trabazo, R., Lucía, A., López-de-Lara, D., Moreno-Aznar, L.A., \& Rodríguez-Martínez, G. (2020). Ejercicio físico como «medicina» en enfermedades crónicas durante la infancia y la adolescencia. Anales de Pediatría. doi:10.1016/j. anpedi.2020.01.010.

2. Arocha, J.I. (2019). Sedentarismo, la enfermedad del siglo XXI. Clínica e Investigación en Arteriosclerosis. doi: 10.1016/j.arteri.2019.04.004

3. Arteaga, A., Bustos, P., Soto, R., Velasco, N., \& Amigo, H. (2010). Actividad física y su asociación con factores de riesgo cardiovascular: Un estudio en adultos jóvenes. Revista médica de Chile, 138(10), 1209-1216.

4. Báez, L., Blanco, M., Bohórquez, R., Botero, L., Cuenca, G., y Roa, N. (2007). Guías colombianas para el diagnóstico y tratamiento de la hipertensión arterial. Revista Colombiana de Cardiología, 13(Supl 1):195206.

5. Bonilla-Arena, E. y Sáez-Torralba, M. E. (2014). Beneficios del ejercicio físico en el adulto. RqR Enfermería Comunitaria, 2 (4), 21-30.

6. Capece, M., Creta, M., Calogero, A., La Rocca, R., Napolitano, L., Barone, B., Sica, A., Fusco, F., Santangelo, M., Dodaro, C., Sagne1li, C., Carlomagno, N., Crocetto, F., Califano, G., Mangiapia, F., \& Longo, N. (2020). Does Physical Activity Regulate Prostate Carcinogenesis and Prostate Cancer Outcomes? A Narrative Review. International Journal of Environmental Research and Public Health, 17(4), 1441. https://doi.org/10.3390/ijerph17041441

7. Caro, J., Navarro, I., Romero, P., Lorente, R. I., Priego, M. A., Martínez-Hervás, S., ... \& Ascaso, J. F. (2013). Efecto metabólico del ejercicio físico regular en la población sana. Endocrinología y Nutrición, 60(4), 167-172.

8. Chávez Cevallos, E., Fernández Lorenzo, A., Rodríguez Torres, Á. F., Gómez Díaz, M. G., \& Sánchez Cordova, B. (2017). Intervención desde la actividad física en mujeres hipertensas de la tercera edad. Revista Cubana de Investigaciones Biomédicas, 36(1), 1-10.

9. Crespo-Salgado, J. J., Delgado-Martín, J. L., Blanco-Iglesias, O., y Aldecoa-Landesa, S. (2015). Guía básica de detección del sedentarismo y recomendaciones de actividad física en atención primaria. Atención Primaria, 47(3), 175-183. doi: 10.1016/j.aprim.2014.09.004

10. Danet, A., Prieto-Rodríguez, M.A., Gamboa-Moreno, E., Ochoa-deRetana-Garcia, L., \& March Cerdà, J.C. (2016). La formación entre iguales para pacientes con diabetes mellitus 2 . Una evaluación cuantitativa y cualitativa en el País Vasco y Andalucía. Atención Primaria, 48(8), 507-517. doi: 10.1016/j.aprim.2015.10.010.

11. Danet, A., Prieto-Rodríguez, M.A., Toro-Cárdenas, S.M., GarridoPeńa, F., Escudero-Carretero, M.J., \& March-Cerdà, J.C. (2020). Impacto diferencial, necesidades heterogéneas. La formación entre iguales para mejorar la salud y los hábitos de vida de los pacientes crónicos. Atención Primaria, 52 (2), 112-121. doi: 10.1016/j.aprim.2018.09.020
12. Dirección General de Tráfico (2018). Seguridad vial. Estadisticas e indicadores. Parque de vehículos. Tablas estadisticas. Madrid:DGT. Recuperado de http://www.dgt.es/es/seguridad-vial/estadisticas-e-indicadores/ parque-vehiculos/tablas-estadisticas/

13. Jo, H., Kim, J.-Y., Jung, M.-Y., Ahn, Y.-S., Chang, S.-J., \& Koh, S.B. (2020). Leisure Time Physical Activity to Reduce Metabolic Syndrome Risk: A 10-Year Community-Based Prospective Study in Korea. Yonsei Medical Journal, 61(3), 218-228. https://doi.org/10.3349/ ymj.2020.61.3.218

14. Lamotte, M. (2016). Factores de riesgo cardiovascular y actividad física EMC-Kinesiterapia-Medicina Física, 37(2), 1-7.

15. Leiva, A. M., Martínez, M. A., Cristi-Montero, C., Salas, C., RamírezCampillo, R., Díaz Martínez, X., ... \& Celis-Morales, C. (2017). El sedentarismo se asocia a un incremento de factores de riesgo cardiovascular y metabólicos independiente de los niveles de actividad física. Revista médica de Chile, 145(4), 458-467.

16. López Sánchez, G. F., Ibáńez Ortega, E. J., \& Díaz Suárez, A. (2019) Efectos de un programa de actividad física vigorosa en la tensión arterial y frecuencia cardiaca de escolares de 8-9 años. SPORT TK: Revista Euroamericana de Ciencias del Deporte, 8(1), 73-80.

17. Mahecha, S.M. (2019). Recomendaciones de actividad física. Revista de Nutrición Clínica y Metabolismo, 2(2): 44-54. doi: https://doi. org/10.35454/ rncm.v2n2.006

18. Martos, M. J., Pozo, C., \& Alonso, E. A. (2010). Enfermedades crónicas y adherencia terapéutica: relevancia del apoyo social. Almería: Editorial Universidad de Almería.

19. Ministerio de Sanidad, Consumo y Bienestar Social (2017). Encuesta Nacional de Salud (ENSE), España 2017. Serie informes monográficos. Madrid. Recuperado de https://www.mscbs.gob.es/estadEstudios/estadisticas/encuestaNacional/encuestaNac2017/ACTIVIDAD_FISICA.pdf

20. Ministerio de Sanidad, Consumo y Bienestar Social (2014). Encuesta Europea de Salud en España (EESE) 2014. Madrid. Recuperado de https://www.mscbs.gob.es/estadEstudios/estadisticas/EncuestaEuropea/pdf/EESE14_inf.pdf

21. Nuzum, H., Stickel, A., Corona, M., Zeller, M., Melrose, R. J., \& Wilkins, S. S. (2020). Potential Benefits of Physical Activity in MCI and Dementia. Behavioural Neurology, 2020. https://doi. org $/ 10.1155 / 2020 / 7807856$

22. Organización Mundial de la Salud (OMS) (2018b). Informe sobre la situación mundial de las enfermedades no transmisibles. Ginebra. Recuperado de http://www.who.int/es/news-room/fact-sheets/detail/noncommunicable-diseases

23. Organización Mundial de la Salud (OMS) (2019). Estrategia mundial sobre régimen alimentario, actividad fisica y salud. Ginebra. Recuperado de https://www.who.int/dietphysicalactivity/factsheet_inactivity/es/ 
24. Organización Mundial de la Salud (OMS) (2010). Recomendaciones mundiales sobre actividad física para la salud. Ginebra. Recuperado de https:// apps.who.int/iris/bitstream/handle/10665/44441/9789243599977_ spa.pdf?ua $=1$

25. Organización Mundial de la Salud (OMS) (2018a). Obesidad y sobrepeso. Ginebra. Recuperado de https://www.who.int/es/news-room/factsheets/detail/obesity-and-overweight

26. Orozco-Gómez, A. M., y Castiblanco-Orozco, L. (2015). Factores psicosociales e intervención psicológica en enfermedades crónicas no transmisibles. Revista colombiana de psicologia, 24(1), 203-217. doi: 10.15446/rcp.v24n1.42949

27. Pate, R. R., Pratt, M., Blair, S. N., Haskell, W. L., Macera, C. A., Bouchard, C., ... \& Kriska, A. (1995). Physical activity and public health: a recommendation from the Centers for Disease Control and Prevention and the American College of Sports Medicine. Jama, 273(5), 402-407. doi:10.1001/jama.1995.03520290054029

28. Quílez Llopis, P., y García-Galbis, M.R. (2015). Control glucémico a través del ejercicio físico en pacientes con diabetes mellitus tipo 2; revisión sistemática. Nutrición Hospitalaria, 31 (4), 1465-147.

29. Schwaab, B., Kafsack, F., Markmann, E., \& Schütt, M. (2020). Effects of aerobic and anaerobic exercise on glucose tolerance in patients with coronary heart disease and type 2 diabetes mellitus. Cardiovascular Endocrinology \& Metabolism, 9(1), 3-8. https://doi.org/10.1097/XCE.0000000000000188

30. Subirats-Bayego, E., Subirats-Vila, G. y Soteras-Martínez, I.(2012). Prescripción de ejercicio físico: indicaciones, posología y efectos adversos. Medicina Clínica, 138(1), 18-24. doi: https://doi.org/10.1016/j. medcli.2010.12.008

31. Tobar, S., y Marchiori, P. (2018). Mercosur Frente a las Enfermedades Crónicas No Transmisibiles (ECNT). Revista MERCOSUR de políticas sociales, 2, 90-111. doi: 10.28917/ism.2018-v2-90

32. Vélez-Álvarez, C., y Vidarte Claros, J.A. (2016). Efecto de un programa de entrenamiento físico sobre condición física saludable en hipertensos. Revista Brasileira de Geriatría y Gerontología, 16(2), 277-288. doi: https://doi.org/10.1590/1809-98232016019.140168 